\title{
Invariant Lagrange Submanifolds of Dissipative Systems
}

\author{
A. Agrachev *
}

\begin{abstract}
We study smooth solutions of modified Hamilton-Jacobi equations $H\left(\frac{d u}{d q}, q\right)+\alpha u(q)=0, q \in M$, on a compact manifold $M$.
\end{abstract}

Let $M$ be a compact Riemannian manifold of class $C^{k}, k \geq 2$, with the Riemannian structure $(\xi, \eta) \mapsto\left\langle I_{q}^{-1} \xi, \eta\right\rangle, \xi, \eta \in T_{q} M, q \in M$, where $I_{q}: T_{q}^{*} M \rightarrow T_{q} M$ is a self-adjoint linear map such that the quadratic form $z \mapsto\left\langle z, I_{q} z\right\rangle, z \in T_{q}^{*} M$, is positive definite.

Let $V \in C^{k}(M)$ and $\omega$ be a closed differential 1-form on $M$ of class $C^{k}$ such that $\nabla \omega=0$, where $\nabla \omega$ is the covariant derivative of $\omega$. We consider the Hamiltonian function $H \in C^{k}\left(T^{*} M\right)$ defined by the formula:

$$
H(z)=\frac{1}{2}\left\langle I_{q}\left(z+\omega_{q}\right), z+\omega_{q}\right\rangle+V(q), \quad z \in T_{q}^{*} M
$$

Let $\vec{H}$ be the Hamiltonian vector field on $T^{*} M$ associated to $H$ and $\ell$ be the "vertical" Euler vector field of the vector bundle $T^{*} M \rightarrow M$. In local coordinates, $z=(p, q), p, q \in \mathbb{R}^{n}, T_{q}^{*} M=\left(\mathbb{R}^{n}, q\right)$,

$$
\begin{array}{r}
H(p, q)=\frac{1}{2}(p+\omega(q))^{*} I_{q}(p+\omega(q))+V(q), \\
\vec{H}(p, q)=\sum_{i}\left(\frac{\partial H}{\partial p^{i}} \frac{\partial}{\partial q^{i}}-\frac{\partial H}{\partial q^{i}} \frac{\partial}{\partial p^{i}}\right), \ell(p, q)=\sum_{i} p^{i} \frac{\partial}{\partial p^{i}} .
\end{array}
$$

*SISSA, Trieste and MIAN, Moscow 
We study the dissipative system $\dot{z}=\vec{H}(z)-\alpha \ell(z)$, where $\alpha$ is a positive constant. It is not hard to see that any bounded trajectory of this system is contained in the set

$$
B_{H} \stackrel{\text { def }}{=}\left\{z \in T^{*} M: H\left(z-\omega_{\pi(z)}\right) \leq \max _{q \in M} H\left(0_{q}\right)\right\},
$$

where $0_{q}$ is the origin of $T_{q}^{*} M$ and $\pi: T^{*} M \rightarrow M, \pi\left(T_{q}^{*} M\right)=q$.

Given $z \in T^{*} M$, we denote by $\rho(z)$ the maximal eigenvalue of the symmetric operator

$$
\xi \mapsto \mathfrak{R}\left(\xi, I_{q} z\right) I_{q} z+\left(\nabla_{q}^{2} V\right) \xi, \quad \xi \in T_{q} M,
$$

where $\mathfrak{R}$ is the Riemannian curvature. Finally, we set

$$
r=\max \left\{\rho(z): z \in B_{H}\right\}
$$

Let $\Omega^{\alpha}$ be the set of all absolutely continuous curves $\gamma:[0,+\infty) \rightarrow M$ such that the integral $\int_{0}^{+\infty} e^{-\alpha t}\left\langle I_{\gamma(t)}^{-1} \dot{\gamma}(t), \dot{\gamma}(t)\right\rangle d t$ converges. We introduce the discounted action functional

$$
\mathfrak{I}_{\alpha}(\gamma)=\int_{0}^{+\infty} e^{-\alpha t}\left(\frac{1}{2}\left\langle I_{\gamma(t)}^{-1} \dot{\gamma}(t), \dot{\gamma}(t)\right\rangle-V(\gamma(t))+\left\langle\omega_{\gamma(t)}, \dot{\gamma}(t)\right\rangle\right) d t, \quad \gamma \in \Omega_{\alpha} .
$$

Theorem 1 Let $u(q)=-\inf \left\{\mathfrak{I}_{\alpha}(\gamma): \gamma \in \Omega_{\alpha}, \gamma(0)=q\right\}, q \in M$. If $r \leq 0$ or $0<r<\frac{\alpha^{2}}{4}$ and $k<\frac{2}{1-\sqrt{1-\frac{4 r}{\alpha^{2}}}}$, then:

- $u \in C^{k}(M)$ and the map $(H, \alpha) \mapsto u$ is continuous in the $C^{2}$-topology.

- The function $u$ satisfies the modified Hamilton-Jacobi equation $H(d u)+\alpha u=0$ and $\left\{d_{q} u: q \in M\right\} \subset T^{*} M$ is an invariant submanifold of the system $\dot{z}=\vec{H}(z)-\alpha \ell(z)$.

- There exists a containing 0 neighborhood $\mathcal{O}$ of $u$ in $C^{2}(M)$ such that $\forall v_{0} \in \mathcal{O}$ the classical solution $v_{t}$ of the Cauchy problem $\frac{\partial u_{t}}{\partial t}+H\left(d u_{t}\right)+$ $\alpha u_{t}=0, u_{0}=v_{0}$, is defined for all $t \geq 0$ and $\left\|d v_{t}-d u\right\|_{C^{1}} \rightarrow 0$ as $t \rightarrow+\infty$ with the exponential convergence rate. 
Remark. Theorem 1 is applied to the Hamiltonians in $\mathbb{R}^{n} \times \mathbb{R}^{n}$ of the form

$$
H(p, q)=\frac{1}{2}|p+a|^{2}+V(q),
$$

where $a \in \mathbb{R}^{n}$ is a constant vector and $V$ is a smooth periodic potential. Then

$r$ is the maximum of the eigenvalues of the matrices $\frac{d^{2} V}{d q^{2}}, q \in \mathbb{R}^{n}$. If $r<\frac{\alpha^{2}}{4}$, then the equation $\frac{1}{2}\left|\frac{d u}{d q}+a\right|^{2}+V(q)+\alpha u=0$ has a periodic $C^{k}$-solution $u$, where $k$ is maximal integer that is strictly smaller than $\frac{2}{1-\sqrt{1-\frac{4 r}{\alpha^{2}}}}$. Moreover, $\left\{\left(\frac{d u}{d q}, q\right): q \in \mathbb{R}^{n}\right\}$ is an invariant submanifold of the system

$$
\dot{q}=p+a, \quad \dot{p}=-\frac{d V}{d q}-\alpha p
$$

The proof of Theorem 1 can be derived from [1] and [2]. Indeed, Theorem 1 is an improvement of results of paper [1]. The improvement concerns more general Hamiltonians (nonzero forms $\omega$ are available), better smoothness of $u$, and stability properties. One can check that $\omega$ does not affect the canonical connection and the curvature operators; hence more general Hamiltonians do not require essential changes in the proof.

The better smoothness and stability follow from [2, Th.4.1]. Indeed, Prop. 1 in [1] implies that $\left\{d_{q} u: q \in M\right\}$ is a normally hyperbolic invariant submanifold (see [2] for the definition) of the flow generated by the vector field $\vec{H}(z)-\alpha \ell(z)$. Moreover, this normally hyperbolic invariant submanifold has zero unstable subbundle and can be actually called the "normally stable" invariant submanifold. Theorem 4.1 in [2] contains the estimate for the smoothness class of the normally hyperbolic invariant submanifold in terms of the Lyapunov exponents while the analysis of the proof of Prop. 1 in [1] gives explicit estimates for the Lyapunov exponents in terms of $r$ and $\alpha$.

\section{References}

[1] A. Agrachev, Well-posed infinite horizon variational problems on a compact manifold, arXiv:0906.4433, 23pp.

[2] M. Hirsch, C. Pugh, M. Shub, Invariant manifolds, Lecture Notes in Math., 583. Springer Verlag, 1977, 149pp. 